\title{
BP-PP-4-4
}

\section{Surgical resection after gemcitabine, cisplatin, and nab-paclitaxel chemotherapy for locally advanced cholangiocarcinoma: A retrospective single-center study}

\author{
Hye Jung $\mathrm{CHO}$, Sung Hoon $\mathrm{CHOl}^{*}$
}

Department of Surgery, CHA Bundang Medical Center, Seongnam, Korea

Introduction: We conducted this study to evaluate the clinical feasibility of the triplet chemotherapy involving gemcitabine, cisplatin, and nab-paclitaxel as induction chemotherapy in locally advanced cholangiocarcinoma.

Methods: From October 2019 to October 2020, a total of 15 patients diagnosed with a potentially resectable stage of locally advanced cholangiocarcinoma by a multidisciplinary team and treated with the aforementioned triplet chemotherapy were reviewed.

Results: Three patients were diagnosed with intrahepatic cholangiocarcinoma, eight patients were diagnosed with perihilar cholangiocarcinoma, and four patients were diagnosed with distal cholangiocarcinoma. All patients showed a partial response during an evaluation following induction triplet chemotherapy. Two patients were not able to undergo surgery because of poor performance status, while 13 patients underwent curative surgical resection. The evaluation of pathologic $\mathrm{T}$ stage showed that all patients had stage T2 disease or less. One patient demonstrated complete remission on final pathology. Five out of 10 patients (50\%) with definite lymph node metastasis during initial imaging study showed negative findings in the final pathologic report. R0 resection was achieved in 12 out of 13 patients (92.3\%). Microscopic lymphovascular or perineural involvement was identified in five patients (38.5\%).

Conclusions: Our result demonstrates a feasible effect of gemcitabine-cisplatin plus nab-paclitaxel as induction chemotherapy on locally advanced cholangiocarcinoma prior to curative surgery. 